\title{
Therapeutic Efficacy and Safety of Oral Desmopressin for Nocturia in the Elderly Patient
}

\author{
Víctor Navalón-Monllor ${ }^{1}$, Felipe Ordoño-Domínguez ${ }^{1}$, Celia Ramada-Calaforra ${ }^{1}$, Yoni Pallás- \\ Costa $^{1}, M^{\mathrm{a}}$ Jesús Segura-Jiménez ${ }^{1}$ and Pedro Navalón-Verdejo ${ }^{{ }^{*}}$
}

${ }^{1}$ Catholic University San Vicente Mártir, Valencia, Spain

Received: May 23, 2017; Accepted: June 15, 2017; Published:June 23, 2017

*Corresponding author: Pedro Navalón-Verdejo, Universidad De Valencia, Spain. E-mail: pedronavalon@yahoo.com

\begin{abstract}
Objective: To evaluate the therapeutic efficacy and safety of using oral desmopressin in the treatment of nocturia in the elderly.

Patients And Method: During the last 8 years we have treated with oral desmopressin (Minurin $® 60 \mathrm{mcg}$ ) 118 elderly males (mean age 75 years) suffering from nocturia (mean of 5 urinations). We prospectively evaluated the micturition volume the night before and after treatment and the number of voids and the time until the first night-time micturition. Periodic analytical studies were conducted in all patients, primarily of plasma sodium levels. To assess the impact of nocturia on quality of life and satisfaction with treatment received, we used the validated nocturia-specific N-QoLquestionnaire.

Results: In all patients there was a decrease in the number of nighttime micturitions from the first week of treatment, none presenting any manifestations of clinical relevance to suggest treatment side effects. No significant laboratory abnormalities were evident either, and the patients experienced significant quality of life improvement.

Conclusions: Treatment with oral desmopressin is an effective and well tolerated measure in the elderly nocturia patient. It provides a significant reduction in nocturnal urine volume compared to baseline, showing a reduction in the number of night-time urinations and a prolongation of uninterrupted sleep to first void. This offers a clear improvement in patients' quality of life, with minimal likelihood of adverse effects, and we believe that its use should be included in the urologist's routine therapeutic measures.
\end{abstract}

Keywords: Desmopressin; Nocturia; Elderly

\section{Introduction}

Although some authors define nocturia as the need to get up to urinate more than three times a night, the International Continence Society defines it as "the situation in which the individual gets up at night one or more times to void". It is one of the most difficult symptoms to diagnose and treat effectively and urologists are faced with it daily. Recent studies show that nocturia is the most frequent symptom of the lower urinary tract, mentioned by more than $70 \%$ of elderly patients, and it also has a profound impact on quality of life [1-3]. The differential diagnosis must also consider various non-urological causes. Likewise, failures in the response to therapy directed to the lower urinary tract should lead to suspecting the existence of other nonurological aetiologies [4].

Nocturia is considered one of the most bothersome lower urinary tract symptoms in both men and women. There are many studies on the prevalence of nocturia according to various assessment criteria, but they all seem to agree that the prevalence of this condition is similar in both sexes and increases with age $[5,6]$. Indeed, most studies agree that this is a situation that mainly affects the elderly patient, but still there is a significant proportion of young patients suffering episodes of nocturia [7].

Studies describe two main mechanisms involved in nocturia; on the one hand increased urinary frequency, mainly related to urological problems, and on the other an increase in urine output [8-10]. In addition, Obstructive Sleep Apnoea (OSA) is related to an increased production of urine, frequently causing a situation of nocturia. This syndrome triggers a complex series of events that lead to increased negative intrathoracic pressure and increased venous blood flow to the heart, which causes atrial enlargement and dilation of the right ventricle. To compensate for this, the heart releases Atrial Natriuretic Peptide (ANP), which inhibits the secretion of Antidiuretic Hormone or Vasopressin (ADH, AVP) and aldosterone, thus increasing diuresis through the effect on glomerular filtration of sodium and water. In addition, hypoxia and hypercapnia that occur in each apnoea stimulate the sympathetic nervous system, which also contributes to the heart receiving a false fluid overload signal. The treatment of OSA through administration of Continuous Positive Airway Pressure (CPAP) prevents airway obstruction, avoiding the chain of events that result in nocturnal polyuria and secondarily nocturia in the patient [10-12].

Under normal conditions, a circadian rhythm of urine production decreases it during sleep and increases it during the waking state. Thus, it has been found that young patients with normal sleep cycles have a more concentrated urine and reduced excretion of sodium, potassium, chloride, bicarbonate and water overnight [8]. Likewise, if we exclude pathological causes, such as infection or obstruction, an important cause of nocturia in 
the elderly population is a circadian rhythm disturbance in the secretion of $\mathrm{ADH}$, with an insufficient concentration of this at night [8].

Desmopressin is a synthetic analogue of the 8-arginine vasopressin that increases the reabsorption of water in the renal tubules, thereby decreasing urine output. With oral administration at night, we seek to reduce night-time urine volume and therefore episodes of nocturia, secondarily normalizing sleep patterns of patients, which obviously improves their quality of life.

With this study we aim to assess the therapeutic efficacy and safety of using oral desmopressin in the treatment of nocturia in the elderly.

\section{Material And Methods}

During the last 8 years (January 2007 to January 2015), after approval by the Ethics Committee of our institution and obtaining informed consent, we treated with oral lyophilized desmopressin (Minurin ${ }^{\circledR} 60 \mathrm{mcg}$ ) 118 males with nocturia with an average of 5 voids overnight (range 4 to 10), aged between 69 and 92 years (mean 75 years).

Excluded from the study were all patients whose nocturia was secondary to a well-defined cause, such as primary polydipsia, multiple sclerosis, suspected diabetes insipidus, urge incontinence, recent onset of medical or surgical treatment of
$\mathrm{BPH}$ and patients with severe heart failure.

All patients received a dose of $60 \mu \mathrm{g}$ of lyophilized desmopressin tablets orally at bedtime. A few weeks before starting treatment, all of them were instructed with basic preventive measures, primarily the desirability of reducing fluid intake during dinner and supine rest with legs elevated for one or two hours before sleep.

Efficacy was evaluated by assessing the night and daytime micturition volume during the pre- and post-treatment week and for another week at 3 months of starting treatment. The number of voids and time to first night-time urination was also assessed.

Safety was assessed from a detailed anamnesis and clinical examination primarily aimed at diagnosing potential adverse events, as well as conducting periodic analytical studies, with special emphasis on serum sodium levels. The analytical determinations were made during the week prior to initiation of treatment and at seven days and three months after starting treatment.

To assess the impact of nocturia on quality of life and satisfaction with treatment received, we used the validated nocturia-specific N-QoL questionnaire (Table 1) consisting of 13 questions, each with 5 responses that are rated with values from 0 to 4 . Subjects answered the questionnaire during the week before the start and we repeated it after three months of treatment.

Table: 1 Nocturia-specific quality of life questionnaire (N-QoL)

\section{OVER THE PAST TWO WEEKS HAVING TO WAKE UP TO URINATE.}

\begin{tabular}{|c|r|r|r|r|r|}
\hline 1 & $\begin{array}{r}\text { Has made it difficult for me to concentrate the next } \\
\text { day }\end{array}$ & Every day & Most days & Some days & Rarely \\
\hline 2 & $\begin{array}{r}\text { Has made me feel generally low in energy the next } \\
\text { day }\end{array}$ & Every day & Most days & Some days & Rarely \\
\hline 3 & Has required me to nap during the day & Every day & Most days & Some days & Rarely \\
\hline 4 & Has made me less productive the next day & Every day & Most days & Some days & Rarely \\
\hline 5 & Has caused me to participate less in activities I enjoy & Every day & Most days & Some days & Rarely \\
\hline 6 & $\begin{array}{r}\text { Has caused me to be careful about when or how } \\
\text { much I drink }\end{array}$ & Every day & Most days & Some days & Rarely \\
\hline 7 & Has made it difficult for me to get enough sleep at \\
night & Every day & Most days & Some days & Rarely \\
\hline
\end{tabular}

OVER THE PAST TWO WEEKS, I HAVE BEEN ...

Concerned that I am disturbing others in the house because of having to get up at night to urinate
Extremely
Moderately

A little bit

Not at al 


\begin{tabular}{|c|c|c|c|c|c|c|}
\hline 9 & $\begin{array}{c}\text { Preoccupied about having to get up at night to } \\
\text { urinate }\end{array}$ & All the time & $\begin{array}{l}\text { Most of the } \\
\text { time }\end{array}$ & $\begin{array}{l}\text { Some of the } \\
\text { time }\end{array}$ & Rarely & Never \\
\hline 10 & $\begin{array}{l}\text { Worried that this condition will get worse in the } \\
\text { future }\end{array}$ & Extremely & Quite a lot & Moderately & A little bit & Not at all \\
\hline 11 & $\begin{array}{l}\text { Worried that there is no effective treatment for this } \\
\text { condition (having to get up at night) }\end{array}$ & Extremely & Quite a lot & Moderately & A little bit & Not at all \\
\hline 12 & $\begin{array}{l}\text { Overall, how bothersome has getting up at night to } \\
\text { urinate been during the past two weeks? }\end{array}$ & Not at all & A little bit & Moderately & Quite a bit & Extremely \\
\hline 13 & Overall, I would rate my quality of life to be ... & Very good & Good & Fair & Poor & Very poor \\
\hline
\end{tabular}

Regarding the statistical analysis of the results, the data are expressed as mean, standard deviation and range. The analysis was performed using the SPSS 19 statistical package (SPSS Inc., Chicago, IL). When it was necessary to compare between means, the Student $\mathrm{t}$ test was used, considering statistical significance to be when $\mathrm{p}<0.05$. When we needed to compare qualitative aspects, we used the chi-square test.

Table 2: List of results obtained Mean (DE)

\begin{tabular}{|c|c|c|c|c|c|c|}
\hline & (A) Week pre & $p(A-B)$ & (B) Week post & $p(B-C)$ & (C) 3 months post & $p(A-C)$ \\
\hline Daily urine volume & $1758(325)$ & ns & 1801 (331) & ns & $1796(350)$ & ns \\
\hline Night urine volume & $630(105)$ & $<0.0001$ & $480(101)$ & ns & 497 (97) & $<0.0001$ \\
\hline No. urinations & $5.2(2.7)$ & $<0.0001$ & $3.1(1.9)$ & ns & $3.3(2.0)$ & $<0.0001$ \\
\hline Time 1st urination & 141 (27) & $<0.0001$ & $198(35)$ & ns & $197(43)$ & $<0.0001$ \\
\hline Natremia & $140.2(4.2)$ & $<0.02$ & $139.1(3.9)$ & ns & $138.7(4.4)$ & $<0.005$ \\
\hline Na 125-130 mEq/L & 2 & ns & 5 & ns & 4 & ns \\
\hline NQoL-1 & $22.3(5.1)$ & & & & $16.4(6.3)$ & $<0.0001$ \\
\hline NQoL-2 & $15.6(3.2)$ & & & & $12.4(3.0)$ & $<0.0001$ \\
\hline NQoL-3 & $3.1(1.2)$ & & & & $2.6(1.1)$ & $<0.005$ \\
\hline
\end{tabular}

(A) week pre-treatment. (B) Week post-treatment. (C) three months post-treatment. p (A-B) degree of statistical significance between A and B. p (B-C) degree of statistical significance between B and C. p (A-C) degree of statistical significance between A y C. "ns": not significant. 
None of the patients had symptoms attributable to plasma hyposmolarity (disorientation, lethargy, apathy, nausea, anorexia, etc.) that would require the treatment to be discontinued.

Regarding the impact of nocturia on quality of life and satisfaction with treatment received, we have divided the validated nocturia-specific N-QoL questionnaire into three parts. The first part (NQoL-1), which assesses the degree of sleepfatigue caused by nocturia, reached a score of $22.3 \pm 5.1$ before treatment and was significantly lower ( $p<0.0001$ ) at three months of treatment with an average score of $16.4 \pm 6.3$. There were also significant differences $(p<0.0001)$ in the second part of the questionnaire (NQoL-2), which evaluates the troubleconcern the patient presents, going from $15.5 \pm 3.2$ to $12.4 \pm 3.0$ points. The third part of the questionnaire (NQoL-3) estimating quality of life in general was $3.1 \pm 1.2$ and dropped significantly ( $p<0.005)$ to a score of $2.6 \pm 1.1$ at 3 months after the start of therapy (Table 2).

\section{Discussion}

Nocturia is one of the most common lower urinary tract symptoms, affecting up to $72 \%$ of the elderly, according to various studies. It is also one of the most difficult symptoms to alleviate $[3,4]$.

Regarding its aetiology, there are multiple causes related to nocturia in the elderly. Among them are alterations in circadian $\mathrm{ADH}$ secretion, which normally increases during sleep leading to increased water reabsorption in the renal tubules. This circadian rhythm seems not to exist in older adults with nocturia, in which levels of ADH during sleep hours are undetectable, thereby producing increased Nocturnal Urine Volume (NUV) and the need to evacuate this increase in urine output during the night. This usually determines the need to get up to urinate during the night hours, negatively affecting normal sleep patterns and causing a worse quality of sleep with the consequent impact on quality of life and the patient's nocturnal rest [13-15].

In addition, the need to get up at night to urinate may cause domestic accidents, with frequent falls during the night increasing the risk of fractures and therefore leading to increased morbidity and mortality in the elderly patient [16].

All these factors make therapeutic action appropriate for these patients, not only to minimize the inconvenience caused by nocturia, thereby improving quality of life, but also to reduce the risk of associated injury.

The initial treatment of nocturia will mainly depend on its cause. Thus, when secondary to morphological or functional abnormalities of the lower urinary tract, this requires their correction, either surgically or pharmacologically. Restricting fluids at night and diuretics during the day is a quite effective measure in many cases [17].

It is a known fact that severe nocturia in a significant proportion of elderly men with lower urinary tract symptoms is caused by the lack of nocturnal secretion of ADH that conditions nocturnal polyuria and natriuresis. This reasoning clearly justifies the therapeutic approach for these patients with nocturnal ADH when they do not respond to conventional treatments.

Oral desmopressin is a synthetic analogue of 8-arginine vasopressin that increases water reabsorption in the renal tubules, decreasing urine output; it is the only medical treatment with confirmed effectiveness [15]. However, in Spain its use has been approved only for the treatment of enuresis and diabetes insipidus, with no mention on its summary of product characteristics of the indication for treatment of nocturia.

Numerous studies show that after this treatment sleep quality increases and a significant reduction in nocturia episodes is achieved [18-20]. Thus, in our study there was a significant decrease in nocturnal urine volume compared to baseline, both during the first week and the control performed within three months of initiation of therapy, which led to a decrease in the number of nocturnal voids and consequently a statistically significant increase in the time to first micturition. These last two aspects (number of night-time micturitions and NUV) are considered by many authors the key points of treatment effectiveness [21]. Likewise, prolonging the time until the first micturition is considered a very important quality of life factor, as in the first half of sleep there is an increase in the proportion of so-called "delta sleep", which represents the sleep phase that most contributes to the feeling of having slept well [22]. This type of sleep is at its maximum in adolescence and decreases with age, reaching its nadir in senescence when it is replaced by a greater amount of sleep in the Rapid Eye Movement (REM) phase. Insomnia therapy is therefore currently directed on the one hand toward avoiding circumstances that prevent the achievement of enough delta sleep, such as night pain or nocturia, which subdivide sleep and inhibit achieving deep sleep and on the other hand looking for neuromodulator drugs that increase the percentage of delta sleep to contribute to quality sleep $[22,23]$. Indeed, in the patients in our study we found that with the administration of oral desmopressin there was prolongation of uninterrupted sleep until the first night-time urination, which occurred at about 3,5 hours as opposed to 2 hours at baseline. This led to a clear improvement in the quality of life questionnaire score.

Regarding the assessment of the quality of life, although there are several questionnaires described in the literature, we used the validated nocturia-specific N-QoL questionnaire described by Abrams which is divided into 3 parts [14]. The first part (NQoL1 ) assesses the degree of sleep-fatigue caused by nocturia. The second part of the questionnaire (NQoL-2) assesses the troubleconcern that the patient experiences and the third part of the questionnaire (NQoL-3) estimates general quality of life (Table 1). In our study, we found a significant improvement in all scores on the questionnaire compared to baseline scores.

The safety of using desmopressin is similar to that of placebo except for the risk of developing hyponatremia when very high doses are used, such as 100-400 $\mu$ g orally $[15,24,25]$. Thus, due to the adverse effects that can occur, some authors advocate its use with caution, initiating therapy with desmopressin at low doses and gradually increasing the dose according to patient 
tolerance [26]. However, if administered at appropriate doses, the onset of severe hyponatremia is exceptional. Thus, in our experience, although there was a clear tendency to hyponatremia compared to baseline figures, these were kept within the normal range, with average figures of serum sodium around $139 \mathrm{mEq} / \mathrm{L}$ at both one week and three months after starting treatment. Only 5 patients one week after administration of desmopressin and 4 patients three months later presented figures indicating mild hyponatremia (between 125 - $130 \mathrm{mEq} / \mathrm{L}$ ). In addition, this fact lacked clinical significance, since there was no evidence of the presence of clinical manifestations (disorientation, lethargy, apathy, nausea, anorexia, etc.) that would have forced the reduction of the dose of desmopressin or the suspension of treatment.

In the long term treatment of nocturia, it has been observed that the administration of desmopressin for a year in elderly patients does not affect the secretion of ADH; however, sodium gradually decreasing may induce hyponatremia even in patients who had no problems initially. Therefore, in long-term treatment with desmopressin, serum sodium concentration should be assessed at least every 6 months [27]. If the situation of hyponatremia or the onset of symptoms attributable to plasma hyposmolarity is confirmed, simple discontinuation of therapy reverses the situation immediately $[26,27]$.

\section{Conclusions}

Treatment with desmopressin administered orally is an effective and well tolerated measure for nocturia in the elderly patient, providing a significant reduction in nocturnal urine volume compared to baseline. It shows a reduction in the number of night-time urinations and a prolongation of uninterrupted sleep to first void, offering a clear improvement in patients' quality of life, with minimal likelihood of adverse effects. We believe that its use should be included in the urologist's routine therapeutic measures.

\section{References}

1. Abrams P, Cardozo L, Fall M, Griffiths D, Rosier P, Ulmsten U, et al The standardisation of terminology of lower urinary tract function: report from the Standardisation Sub-committee of the International Continence Society. Neurourol Urodyn. 2002;21(2):167-178.

2. Van Kerrebroeck P, Abrams P, Chaikin D, Donovan J, Fonda D, Jackson $\mathrm{S}$. The standardisation of terminology in nocturia: report from the Standardisation Sub-committee of the International Continence Society. Neurourol Urodyn. 2002;21(2):179-183.

3. Van Doorn B, Bosch JL. Nocturia in older men. Maturitas. 2012;71(1):812. doi: 10.1016/j.maturitas.2011.10.007.

4. Mark D, Walters MD, Mickey M, Karram M. Uroginecología y cirugía reconstructiva de la pelvis. 3rd Edition. Madrid: Elsevier Mansson. 2008.

5. Irwin DE, Milsom I, Hunskaar S, Reilly K, Kopp Z, Herschorn S, et al. Population-based survey of urinary incontinence, overactive bladder, and other lower urinary tract symptoms in five countries: results of the EPIC study. Eur Urol. 2006;50(6):1306-1314. doi: 10.1016/j. eururo.2006.09.019
6. Fitzgerald MP, Litman HJ, Link CL, McKinlay JB, BACH Survey Investigators. The association of nocturia with cardiac disease, diabetes, body mass index, age and diuretic use: results from the BACH survey. J Urol. 2007;177(4):1385-1389. doi: 10.1016/j. juro.2006.11.057

7. Bosch JL, Weiss JP. The prevalence and causes of nocturia. J Urol. 2010;184(2):440-446. doi: 10.1016/j.juro.2010.04.011

8. Chasens ER, Umlauf MG. Nocturia: a problem that disrupts sleep and predicts obstructive sleep apnea. Geriatr Nurs. 2003;24(2):76-81,105.

9. Zachoval R, Krhut J, Sottner O, Hanuš T, Martan A, Horčička L, et al. Nocturia, incidence, ethiology, diagnostics. Ceska Gynekol. 2013;78(6):566-572.

10. Umlauf MG, Chasens ER. Sleep disordered breathing and nocturnal polyuria: nocturia and enuresis. Sleep Med Rev. 2003;7(5):403-411.

11.Umlauf MG, Chasens ER, Greevy RA, Arnold J, Burgio KL, Pillion DJ. Obstructive sleep apnea, nocturia and polyuria in older adults. Sleep. 2004;27(1):139-144.

12. Lowenstein L, Kenton K, Brubaker L, Pillar G, Undevia N, Mueller ER . The relationship between obstructive sleep apnea, nocturia, and daytime overactive bladder syndrome in women. Am J Obstet Gynecol. 2008;198(5):598.e1-5. doi: 10.1016/j.ajog.2008.02.024

13. Chartier-Kastler E, Davidson K. Evaluation of quality of life and quality of sleep in clinical practice. Eur Urol Suppl. 2007;6(9):576-584. doi: 10.1016/j.eursup.2007.01.006

14. Abraham L, Hareendran A, Mills IW, Martin ML, Abrams P, et al. Development and validation of a quality-of-life measure for men with nocturia. Urology. 2004;63(3):481-486. doi: 10.1016/j. urology.2003.10.019

15. Lose G, Lalos O, Freeman RM, van Kerrebroeck P; Nocturia Study Group. Efficacy of desmopressin (Minirin) in the treatment of nocturia: a double-blind placebo-controlled study in women. Am J Obstet Gynecol. 2003;189(4):1106-1113.

16. Jensen J, Lundin-Olsson L, Nyberg L, Gustafson Y. Falls among frail older people in residential care. Scand J Public Health. 2002;30(1):5461.

17. Lose G, Alling-Moller L, Jennum P. Nocturia in women. Am J Obstet Gynecol. 2001;185:514-521.

18. Abrams P, Andersson KE, Birder L, Brubaker L, Cardozo L, Chapple $\mathrm{C}$, et al. Fourth International Consultation on Incontinence Recommendations of the International Scientific Committee: Evaluation and treatment of urinary incontinence, pelvic organ prolapse, and fecal incontinence. Neurourol Urodyn. 2010;29(1):213240. doi: $10.1002 /$ nau. 20870

19. Oelke M, Bachmann A, Descazeaud A, Emberton M, Gravas S, Michel $\mathrm{MC}$, et al. Guidelines on the management of male lower urinary tract symptoms, including benign prostatic obstruction. EurUrol. 2013;64(1):118-140.

20.Thüroff JW, Abrams P, Andersson KE, Artibani W, Chapple CR, Drake MJ, et al. EAU guidelines on urinary incontinence. Eur Urol. 2011;59(3):387-400. doi: 10.1016/j.eururo.2010.11.021

21. Park HK, Kim HG. Current evaluation and treatment of nocturia. 
Korean J Urol. 2013;54(8):492-498. doi: 10.4111/kju.2013.54.8.492

22. Ordoño Domínguez JF. Efectos positivos de pregabalina en las alteraciones del sueño. En: Vidal A, Moya J, editores. Un enfoque multidisciplinar del dolor. Madrid: Ed. Cerssa. 2010;98-106.

23. Ordoño Domínguez JF. Trastornos del sueño y dolor neuropático. Rev SocEspDolor. 2011;18(1):39-40.

24. Rembratt A, Riis A, Norgaard JP. Desmopressin treatment in nocturia; an analysis of risk factors for hyponatremia. Neurourol Urodyn. 2006;25(2):105-109.
25.Sand PK, Dmochowski RR, Reddy J, van der Meulen EA. Efficacy and safety of low dose desmopressin orally disintegrating tablet in women with nocturia: results of a multicenter, randomized, double-blind, placebo controlled, parallel group study. J Urol. 2013;190(3):958-64. doi: 10.1016/j.juro.2013.02.037

26.Shindel A, Tobin G, Klutke C. Hyponatremia associated with desmopressin for the treatment of nocturnal polyuria. Urology. 2002;60(2):344.

27.Jin MH, Moon du G. Practical management of nocturia in urology. Indian J Urol. 2008;24(3):289-94. doi: 10.4103/0970-1591.42607 